\title{
Generalized Software Requirements to Access Thesauri and Classification Schemes for User-Based Image Collections
}

\author{
Barbara Barnes \\ University Library \\ University of Illinois at Urbana-Champaign \\ Eric H. Johnson \\ CANIS/Community Systems Lab \\ Graduate School of Library and Information Science \\ University of Illinois at Urbana-Champaign \\ Jennifer B. Young \\ Graduate School of Library and Information Science \\ University of Illinois at Urbana-Champaign \\ With Assistance from: \\ Pauline A. Cochrane \\ Graduate School of Library and Information Science \\ University of Illinois at Urbana-Champaign
}

\begin{abstract}
This paper describes proposed schemes for describing and indexing two image collections and requirements for the software that would give the end-user access to thesaurus terms and a graphical display of the faceted classification structures of indexing records. Editorial cartoons comprise one collection, dance videos the other.
\end{abstract}

\section{Introduction}

Indexing for editorial cartoons, as well as for dance videos, has typically overlooked the content of the work. Attempting to index the content or meaning of the images is difficult for both types of collections, because it requires interpretation of meaning on the part of the indexer. Faceted classification schemes can assist the indexer in defining the parameters of the information that needs to be captured.

\section{Classification scheme for editorial cartoons}

Editorial cartoons reflect the nation's culture and history at a specific moment in time. They also provide invaluable insight into the mood of the country as well as illustrate debates and controversies over the years. Thus, it is unfortunate that most editorial cartoons are never indexed - especially by the newspapers and magazines in which they appear. Points of access to the collection are often limited to the cartoonist's name, the newspaper's name, and occasionally 
the date. More specialized collections that are not Internet-based do more indexing than previously mentioned, but they are not widely available. ${ }^{1}$

Interpreting editorial cartoons is another challenge to indexing them. Providing subject access is difficult when it can't be determined what the subject is. An indexer needs to be aware of all allusions that the cartoonist makes in her cartoon. Is the cartoonist quoting William Shakespeare or Dave Barry? And how does that quote pertain to the visual components of the cartoon? Is it Richard Nixon pretending to be Hamlet? Or is it an owl quoting George Bush? To able to accurately determine the subject matter of an editorial cartoon the indexer must follow current events and popular culture. For example, when an indexer checked the original source of a cartoon initially indexed under the broad category "environment", she found that it was actually about Earth Day.

The visual aspects are an important component of a cartoon and are often not indexed. Cartoonists often use well-known images to convey their points. In books on the history of political cartoons, whole chapters are devoted to cartoons using the Lincoln Monument and the Statue of Liberty as a motif. Other images include famous photographs and artwork, as well as movie posters and political symbols.

Figure 1 presents a proposed set of facets for indexing editorial cartoons. Subject Access, Visual Access, and Item Information are the top-level facets. Subject Access is composed of Topic, Person, Place, Event, and Time Period. Visual Access includes Person and Place as well, but also includes Object, Caption and Text.

Captions include any "tag-lines" in the cartoon's panels while the Text of the cartoon includes any other text in the cartoon itself. Including the captions and text allows the cartoon to be searched via full-text. In addition, cartoons are a visual medium and a user may only remember seeing "Nixon Library" but not necessarily the rest of the cartoon.

Person and Place under Visual Access are distinct from Person and Place under Subject Access because the cartoon may be a visual analogy in which the literal representation in the cartoon (Visual Access) differs from what it signifies (Subject Access).

The Item Information facet includes Cartoonist, Form, Syndicate, Date, Publisher, Title (if known), and the Abstract. Writing a short abstract permits the indexer to expand on ideas or concepts in the cartoon. This facet, as suggested by Layne (1994), contains information about the "'birth' of an image," as well as its collection history.

${ }^{1}$ The New Yorker has a Cartoon Bank which indexes cartoons that have appeared in the magazine. A user would have to call the magazine and give the librarian the necessary information as to what the cartoon is about before it is sent to the user. The user does not see the cartoon until it is sent. The Centre for the Study of Cartoons and Caricature at the University of Kent in Canterbury does have a graphical interface to its cartoon collection as well as some indexing, but it is only available to users who travel to the university. 


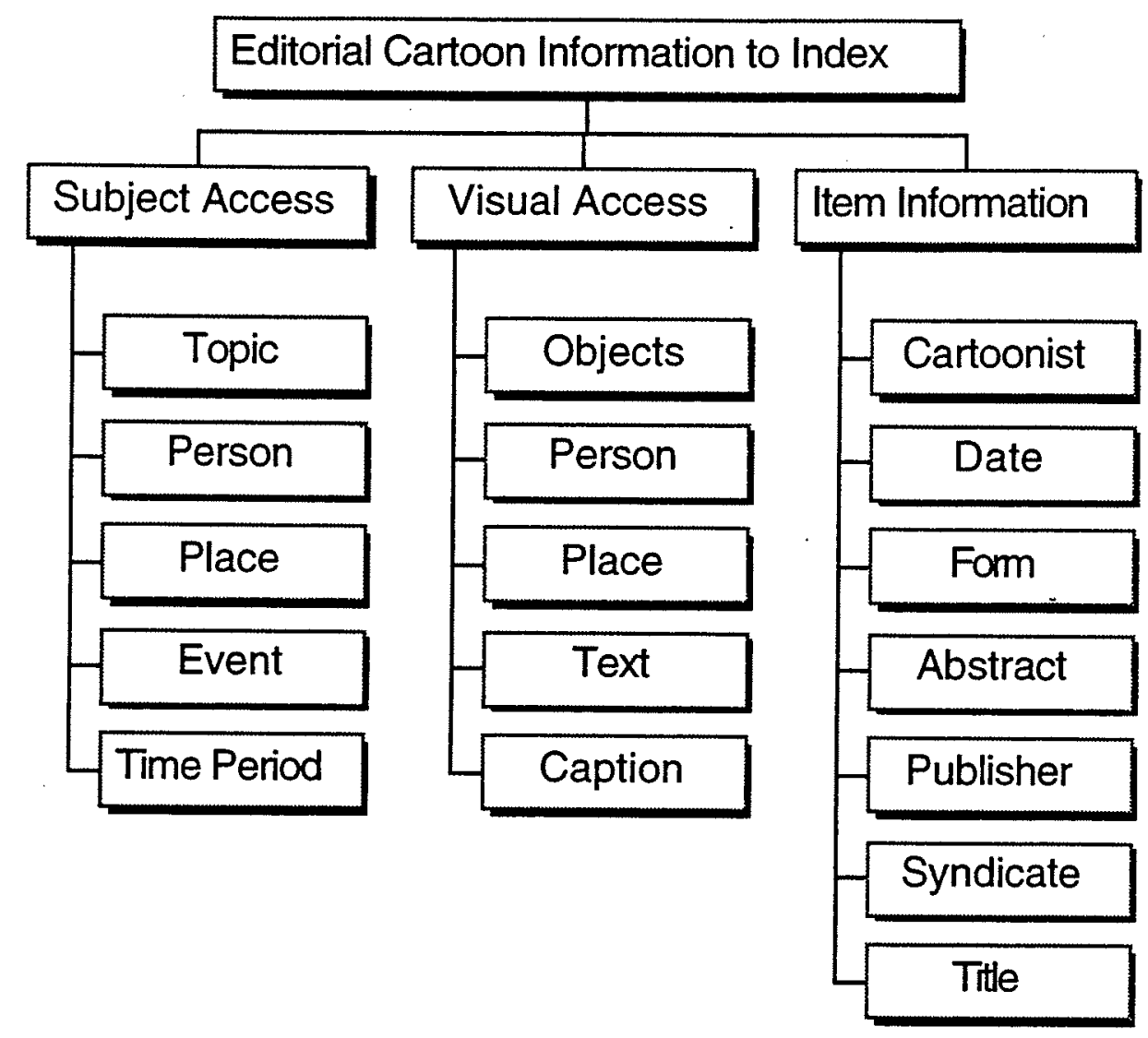

Figure 1. Faceted classification scheme for editorial cartoons.

\section{Guidelines for Indexing editorial cartoons}

$<$ Try to interpret cartoons from the artist's point of view

$<$ Look at the major stories in newspapers of the day to determine current events

$<$ Provide visual access to the cartoon through words

$<$ Include captions

< Write a short abstract of the cartoon's contents or aboutness

〈Choose subject terms, from the thesaurus, for persons, places, events, time period and topical access 
A proposed list of processing rules for extracting all the necessary data for editorial cartoons follows.

\section{Subject access to "aboutness" of cartoon}

Topic: Any subject headings or indexer-created terms from the thesaurus.

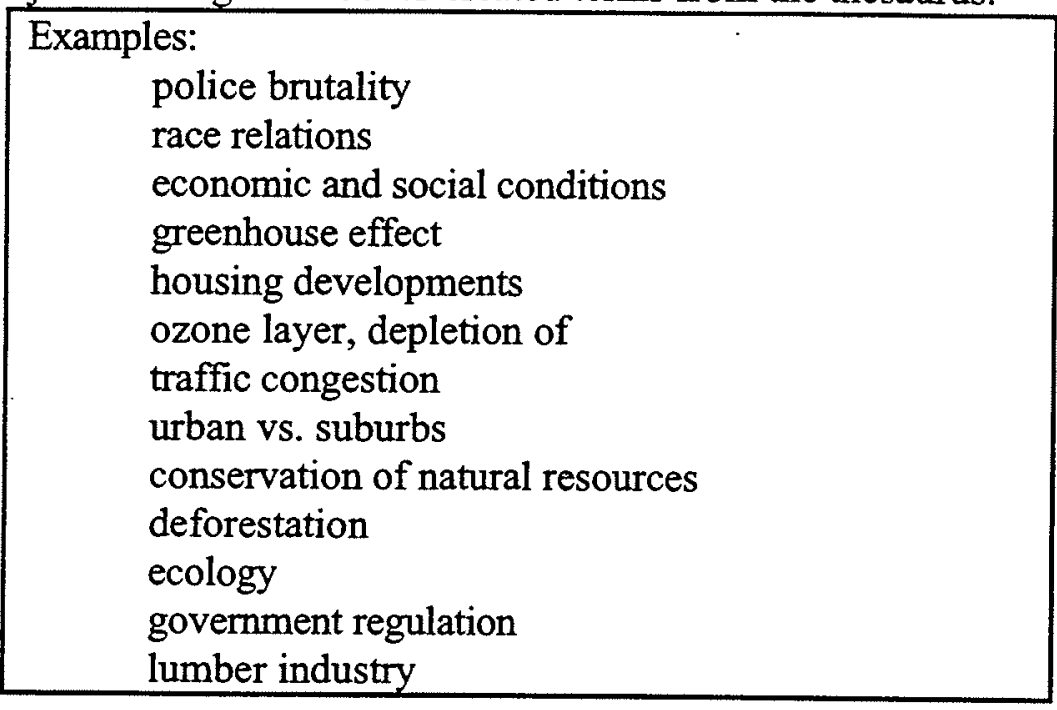

Person: Enter the person's name (last, first) - or as from the name authority file. Examples:

King, Rodney

Nixon, Richard M.

Place: Enter either the place name or type of place.

\begin{tabular}{|l|}
\hline Examples: \\
California \\
Los Angeles \\
Oregon \\
suburbs \\
urban areas \\
forests \\
Iraq \\
Kuwait \\
Pacific Northwest \\
\hline
\end{tabular}

Event: Enter an event

Examples:
campaigns and battles
loggers, unemployment of
Watergate
beating of Rodney King
Earth Day

Time Period: Enter details about the time period. 


\section{Visual access to visual content of cartoon}

Objects: Physical objects in the cartoon itself.

\begin{tabular}{|l|}
\hline Examples: \\
circle of police \\
crescent moon \\
night \\
police brutality \\
game board \\
transportation \\
librarians \\
library \\
patrons \\
tape recorder \\
\hline
\end{tabular}

Caption: Enter here the caption of a cartoon.

\begin{tabular}{l} 
Examples: \\
Nixon Library \\
Regular Library \\
Another Hostage Situation \\
\hline
\end{tabular}

Text: Enter any other text found in the cartoon.

\section{Examples:}

Air conditioner emissions deplete the ozone layer.

Autocracy.

Auto emissions cause Greenhouse Effect.

Avoid public transit: system loses supporters.

Billboard zone: close eyes

Climate heats up: turn on air conditioner.

Person: Enter the person's name (last, first). This facet is used for the people who appear in the cartoon

$$
\text { Example: }
$$

Nixon, Richard M.

Place: Enter either the place name or type of place.

\section{Item information}

Cartoonist: Enter the cartoonist's name (last, first).

Form: Physical Description of the cartoon (color, size, etc) 
Abstract: An indexer-written short abstract about the cartoon.

Examples:
Autocracy: as suburbanites commute to and from
work in the city, they start a chain reaction of
environmental effects.
The LAPD beats itself up by its handling of a
beating of a black man, Rodney King, by white
police, but they'll "get over it."

Publisher: Enter the publisher's (the originating newspaper) information as well as the publication date.

Syndicate: Enter the national syndicate's name here.

Title: Enter the title of the cartoon if available.

The cartoon in Figure 2 commemorated Earth Day 1989.

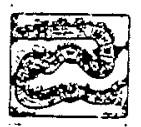

Figure 2. Tom Toles' 1989 Earth Day cartoon.

Toles copyright 1989 The Buffalo News. Reprinted with permission of Universal Press Syndicate. All rights reserved.

Example: Earth Day cartoon

\begin{tabular}{|l|l|}
\hline Cartoonist: & Toles, Tom \\
\hline Date: & $04 / 23 / 1989$ \\
\hline Form: & One-panel cartoon; b\&w; 6 x 7 in. \\
\hline Abstract: & $\begin{array}{l}\text { Autocracy: as suburbanites commute to and from work in the city. } \\
\text { They start a chain reaction of environmental effects. }\end{array}$ \\
\hline Publisher: & Buffalo: Buffalo News, 04/23/89 \\
\hline Syndicate: & Universal Press Syndicate \\
\hline Title: & \\
\hline
\end{tabular}


Example: Earth Day cartoon, cont.

\begin{tabular}{|c|c|}
\hline Topic: & $\begin{array}{l}\text { automobiles } \\
\text { conservation of natural resources } \\
\text { ecology } \\
\text { economic and social conditions } \\
\text { greenhouse effect } \\
\text { housing developments } \\
\text { ozone layer, depletion of } \\
\text { suburban life } \\
\text { suburban sprawl } \\
\text { traffic congestion } \\
\text { urban flight } \\
\text { urban vs. suburbs }\end{array}$ \\
\hline Person: & \begin{tabular}{|l|}
$\begin{array}{l}\text { commuters } \\
\text { suburbanites }\end{array}$ \\
\end{tabular} \\
\hline Place: & $\begin{array}{l}\text { suburbs } \\
\text { urban areas } \\
\end{array}$ \\
\hline Event: & Earth Day \\
\hline Time Period: & $\begin{array}{l}04 / 23 / 1989 \\
\text { Earth Day } \\
\end{array}$ \\
\hline Objects: & $\begin{array}{l}\text { game board } \\
\text { transportation }\end{array}$ \\
\hline Text: & $\begin{array}{l}\text { Air conditioner emissions deplete the ozone layer. } \\
\text { Another new shopping mall: take Pepto Bismol. } \\
\text { Another new subdivision: more demands for new highways. } \\
\text { Autocracy. } \\
\text { Auto emissions cause Greenhouse Effect. } \\
\text { Avoid public transit: system loses supporters. } \\
\text { Billboard zone: close eyes } \\
\text { Buy gasoline: contribute to foreign dependence. } \\
\text { Climate heats up: turn on air conditioner } \\
\text { Flee city: urban deterioration continues } \\
\text { Home! Reflect on the quality of life your car makes possible } \\
\text { Leave new parking garage: another blight on the urban landscape } \\
\text { New highway: woods go down - taxes go up } \\
\text { Radio report of new oil spill } \\
\text { Road repair: lose more time - pay taxes } \\
\text { Smog alert: hold breath } \\
\text { Suburban mall: buy Pepto Bismol } \\
\text { Think about your car payments you can't afford } \\
\text { Traffic jam: lose time }\end{array}$ \\
\hline
\end{tabular}




\section{Classification scheme for dance videos}

Dance materials, in general, have typically been indexed on proper names: the name of the dance and the names of the people involved (e.g., choreographer, composer, principal dancers, dance company). The subjects, or meanings, of the dances have not been well captured. Hanna (1970) explained that social scientists often look for information about the process of dancing (e.g., how decisions are made concerning who should dance or why). Scholars may be interested in studying, for example, how Asian cultures are portrayed in North American productions or how an archetypal character is presented. With the current state of subject access to dance materials, these scholars would have to know in advance which dances contain the subjects they want to study, because the indexing does not include access to the subject or meaning of the dance.

Figure 3 presents a proposed set of facets for indexing dance videos. There are 3 possible layers, or categories, of information in an individual item being indexed: 1) information about the dance being performed (its creators, its form, its subject, gestures, or emotions), 2) information about putting on a production of this dance (the performers, the management and funding, date, etc.), and 3 ) the information about the item itself, e.g., a video (its title, format, etc.).

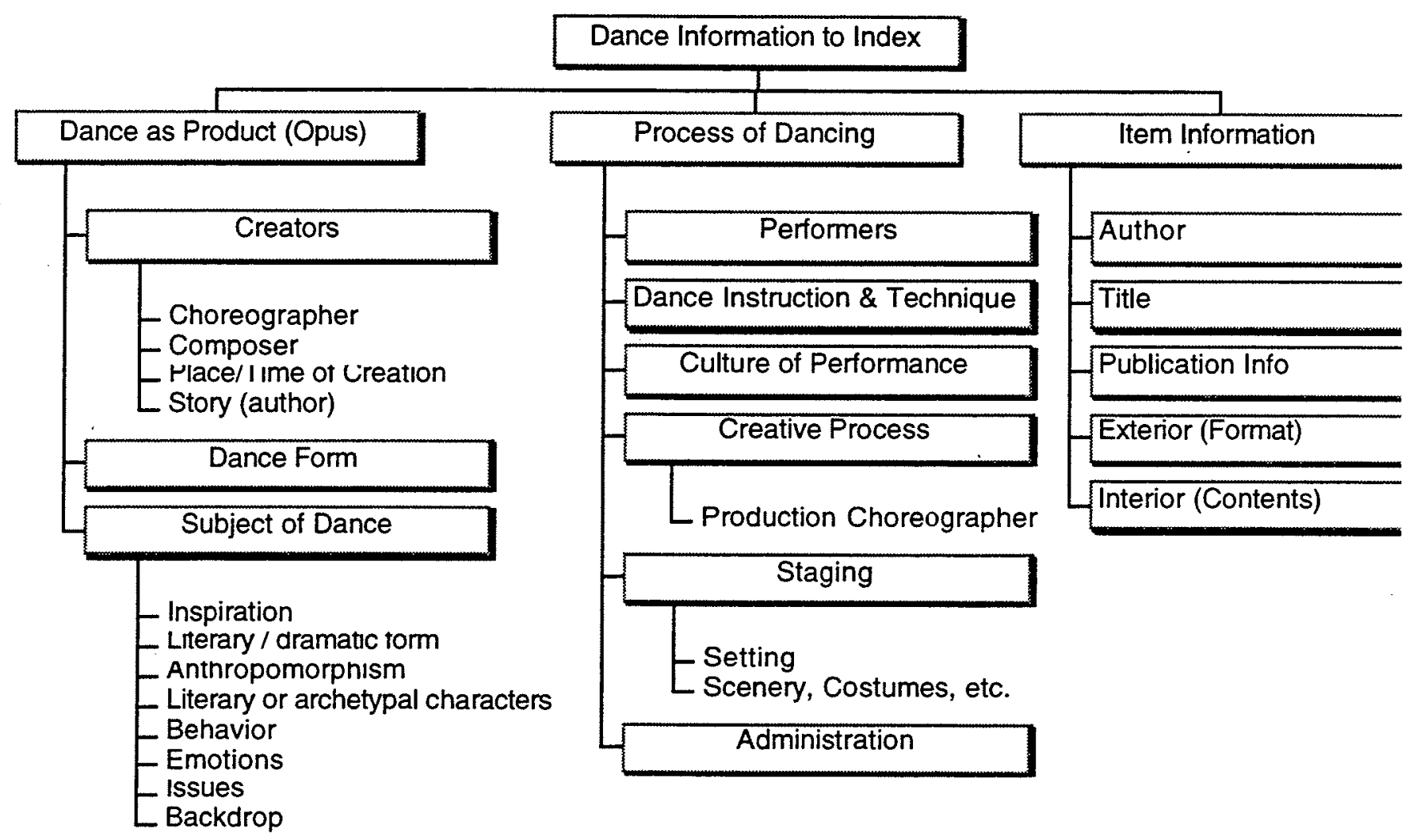

Figure 3. Faceted classification scheme for dance videos. 
Cataloguers of dance materials already cover the groups under major facet 3 quite well. Under major facet 2, "Performers" is quite well covered. In major facet 1 , only "Creators" is very well covered. Because the descriptive catalog record from either OCLC or the New York Public Library is often quite complete (covering the item information in major facet 3 ), we could merely augment the record by attaching the new indexing terms to more completely cover the subject information from major facets 1 and 2.

To define the subject (major facet 1), the indexer must interpret the gestures of the dancers. The enduring power of dance to reach and entertain audiences lies in its ability to portray deep human emotions and life experiences through non-verbal communication. The difficulty for the indexer lies in interpreting these non-verbal stylized movements and translating them into intellectually meaningful terms. This task is difficult enough in dances with well-defined stories, but becomes monumentally difficult in abstract dances, where the main topic of the dance may be only the choreographer's enjoyment of a particular piece of music. Relying on reviews or descriptions by experts can make the process easier and the results more reliable, but admittedly, the process is still difficult and time-consuming. Developing a list of terms for these aspects would be an . important first step for a Dance Thesaurus.

Gestures created in another culture may be impossible for an individual indexer to interpret. As Hanna (1970) explained: "...dance is shaped by the values, attitudes, and beliefs of the people who comprise its 'host' society; it depends on their feeling, thinking, and acting patterns. Thus, the elements of space, rhythm, and dynamics... do not exist apart from the human behavioral processes which produce them."

\section{Guidelines for Indexing Dance Videos:}

A proposed list of processing rules for extracting all the necessary data for a record follow:

\section{Dance as product (OPUS):}

When recording information about a particular dance opus, record all available information in the following areas:

Name of Dance: Enter the official name of the dance (refer to an authoritative referencework). If it is commonly known by another spelling or in another language, make entries for these as well. Creators:

(a) Choreographer:

(b) Composer:

(c) Created: [Country]--[Century]

(d) Story: (author's name, if based on literary work)

Dance Form: Describe the structure of the dance. For staged works, include the applicable descriptor "story" or "plotless."

Examples:
Story ballet
Plotless modern dance
Social Dancing
Kabuki work


Subject of the Dance: Whenever possible, enter subject headings that describe what thedance is about. Whenever possible, the indexer should refer to at least two objective descriptions of the work, in addition to her own perceptions of the meaning. Possible sources of these descriptions include program synopses, reference works, and published descriptions of the choreographer's intentions.

(a) If reliable sources describe a specific inspiration for the work, create a subject heading for it.

\section{Examples:}

Scottish inspiration

Japanese inspiration

Space travel inspiration

(b) For story works, select a subject heading to describe the literary / dramatic form.

$$
\begin{gathered}
\text { Examples: } \\
\text { comedy } \\
\text { drama } \\
\text { tragedy }
\end{gathered}
$$

(c) If the work involves a dance portraying or representing a non-human animal or inanimate object, add a term for each major being represented (singular form).

\begin{tabular}{|l|l|}
\hline Examples: & puppet \\
diamond & ram, longhom \\
doll & rose \\
emerald & ruby \\
flea & \\
\hline
\end{tabular}

(d) If a well-known literary or archetypal character contributes prominently to the meaning of the work, add a term for the character.

\begin{tabular}{|l|l|}
\hline Examples: & peasant \\
bride & pioneer \\
courtesan & preacher (use clergy) \\
clergy & prophet \\
dancer & runaway \\
faun & seer (use prophet) \\
father & shade (use ghost) \\
ghost & Don Quixote \\
groom & Oedipus \\
gypsy & \\
magician & \\
nymph & \\
\hline
\end{tabular}


(e) Add terms for each important identifiable idea represented in the work. These may include expressed emotions that are important to the meaning of the work.

\begin{tabular}{|l|l|}
\hline Examples: & Emotions: \\
Behavior: & aspiration \\
class relations & fear \\
courtship & forgiveness \\
discord & guilt \\
dominance & hope \\
dreaming & love \\
eroticism & optimism \\
forbidden relationships & passion \\
incest & sadness \\
interpersonal attraction & suffering \\
murder & unrequited love \\
opposite sex relations & \\
parent child relations & Issues: \\
rivalry & complexity \\
suicide & ecology \\
& endangered species \\
Backdrop: & homesteading \\
heaven & imprisonment \\
villages & social cohesion \\
\hline
\end{tabular}

\section{Dance as process:}

Performers: If the item represents the work of a production, list the company and any prominently named principal dancers, if available.

Administration: When applicable, make entries related to financing, managing, or decision-making in the dance process.

Creative Process: When applicable (and available), make entries for:

(a) the choreographer for an individual production

(b) other persons or activities related to the process of choreography, composing, or creating the dance performance.

Culture: Make an entry for the culture in which the dance is being performed or prepared. Instruction \& Technique: When applicable, make entries related to the teaching of dance, technique or "how-to" related to dance, nutrition for dancers, or injuries related to dance.

Staging: When applicable, make entries for

(a) the setting of an individual (staged) performance

Examples:

Setting: Place: India

Setting: Place: United States, Time: 19th Century

Setting: Place: Greece, Time: Ancient

(b) any related scenery, costumes, stage management information. 


\section{Item formation}

Author: Enter the name of the author or producer of the video (last, first), if available.

Title: Enter the title of the item being recorded. This information is REQUIRED.

Publisher: Enter the publication date, publisher, and date of publication.

Exterior form: Videorecording.

Interior form: Enter a description of the contents.

\section{Examples: \\ Documentary \\ Performance}

\section{Example: La Bayadere}

\begin{tabular}{|c|c|}
\hline Name of Dance: & La Bayadere \\
\hline Original Creators: & $\begin{array}{ll}\text { Choreographer: } & \text { Petipa, Marius } \\
\text { Composer: } & \text { Minkus, Ludwig } \\
\text { Place: } & \text { Russia } \\
\text { Time: } & \text { 19th Century } \\
\end{array}$ \\
\hline Dance Form: & Story ballet \\
\hline Subject: & 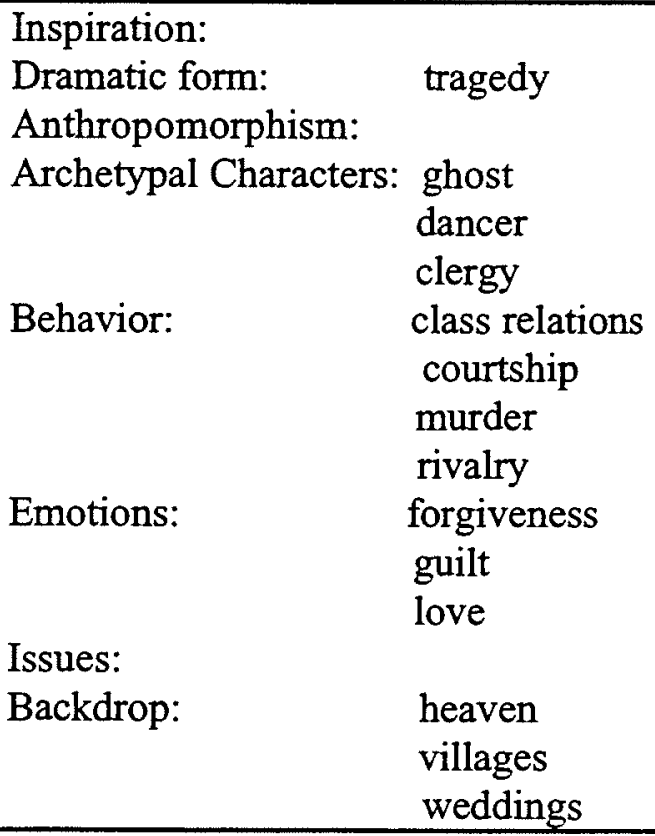 \\
\hline Performers: & $\begin{array}{l}\text { Royal Ballet } \\
\text { Asylnuratova, Altynai } \\
\text { Mukhamedov, Irek } \\
\text { Bussell, Darcey } \\
\text { Kumakawa, Tetsuya } \\
\text { Dowell, Anthony } \\
\end{array}$ \\
\hline Creative Process: & Choreographer: Makarova, Natalia \\
\hline Culture: & $\begin{array}{ll}\text { Place: } & \text { Western } \\
\text { Time: } & \text { Modern } \\
\end{array}$ \\
\hline
\end{tabular}


Barnes, B., Johnson, E., Young, J., \& Cochrane, P. (1998). Generalized software requirements to access thesauri and classification schemes for user-based image collections. 9th ASIS SIG/CR Classification Research Workshop, 1-28. doi:10.7152/acro.v9i1.12742

Proceedings of the 9 th ASIS SIG/CR Classification Research Workshop

Example: La Bayadere, cont.

\begin{tabular}{|l|l|}
\hline Performance Site: & $\begin{array}{l}\text { Place: } \\
\text { Time: }\end{array} \quad \begin{array}{l}\text { England } \\
\text { 20th Century }\end{array}$ \\
\hline Staging: & Setting: $\quad$ India \\
\hline Title: & The Royal Ballet in La Bayadere \\
\hline Publisher: & West Long Branch, NJ: Kultur, 1991 \\
\hline Exterior: & Videorecording \\
\hline Interior: & Performance (Bayadere, La) \\
\hline
\end{tabular}

\section{Thesauri}

Because no one thesaurus exists for describing both the physical attributes of these images and their meaning, the indexer will very likely need to construct a thesaurus by combining terms from different thesauri or from a thesaurus made up of several parts, or even creating a new one. The Art \& Architecture Thesaurus, Iconclass, and the Thesaurus of Graphical Materials have subject content, but each is particular only to the subject matter it covers -- AAT mainly fine art, Iconclass mainly European religious art, and TGM collections of prints and photographs at LC that are primarily part of American history. Dance and cartoon collections need thesauri that cover cartoons, political satire, and social science. These collections require more than any one other thesaurus provides.

Indexing the editorial cartoons began within each category by writing down concepts and ideas as they came. The inclusion of terms in more than one category was necessary when appropriate. Next, LC Subject Headings in the Thesaurus of Graphic Materials was consulted for more uniform terms to use in the index. While the thesaurus was helpful, some of the concepts were not readily apparent or too long to use, because description was only possible with several subdivisions.

Currentness is a problem with LCSH since it is not as up-to-date as the news. Editorial cartoons often contain news items that may not have subject headings or may never have had an appropriate subject heading. The Earth Day cartoon presented particular problems in terms of assigning subject headings. In LCSH, it was difficult to find anything to describe the depletion of the ozone layer, the differences between city life and suburban life and the environmental and economical impact that the suburbs have on urban life. Between the indexer's own terms and the inclusion of the captions and abstract, more complete access has been provided to this cartoon.

For dance videos, the indexer can build on the proper-name indexing already being done in most indexes and add terms for describing the meaning of the dance from Thesaurus of Sociological Indexing Terms, Thesaurus of Psychological Index Terms, and, lastly, LCSH. The advantage of using a thesaurus that already exists is that the structure is already present. The disadvantage is that no one thesaurus contains all the most useful terms, since they were developed for other purposes. 
The name authority file would also need to handle the occurrences of multiple spellings or translations of a name. For example, on one video the dance is spelled "Petrushka," but in many publications, it is spelled "Petrouchka," a problem in transliterating from one alphabet to another. Another video has "L'Apres-midi d'un faune" which is, of course, popularly known as "Afternoon of a Faun." In the ideal database, the searcher could enter either one and still find the correct records; so a name authority file should be attached to the Dance Thesaurus.

\section{Requirements for the retrieval interface to diverse collections of images}

1. The search window should reflect (graphically) the facets of the scheme that were used to index the image.

2. Facets should be displayed in a form that is most appropriate to that facet (e.g., a hierarchy for a thesaurus; a zoomable timeline for a date; a navigable, zoomable map for a place).

3. The searcher should be able to browse the hot-linked authority files, so that any term seen could be used as an actual search term without re-keying it.

4. For each facet, the searcher should be able to see a browse list of the thesaurus terms used in that facet, including the ability to jump to displays for related terms through hypertext.

5. The user should be able to specify more than one term to search for each facet and to combine terms from various facets easily.

6. Most important for searching a faceted database, as the user specifies and re-specifies the search terms they require from multiple facets, the search results should be easy to compare.

7. If the hoped-for term is not in the thesaurus, the interface should guide the searcher to an approximate synonym that is in the thesaurus. Back-up free-text indexes for the collection or synonym dictionaries should be accessible at this stage of the search, without disrupting the search's line of inquiry.

8. If no terms satisfy, the searcher should have a ready mechanism available for suggesting the term to the indexer. This "email" feature helps the indexer / lexicographer understand the research needs for which the collection is being used and to keep up-to-date on searchers' terms.

9. Current catalog records for visual materials may provide some descriptive information for items. It would be useful if these records already in existence either from OCLC, or the New York Public Library or elsewhere, could be easily attached to our records with the new indexing terms.

10. As the Internet will be the way to access these collections, we need to access the image or, in the case of a video, perhaps access a part of the total production. For cartoons, the searcher should be able to retrieve the cartoon on-line during the search. For videos, the searcher should 
Barnes, B., Johnson, E., Young, J., \& Cochrane, P. (1998). Generalized software requirements to access thesauri and classification schemes for user-based image collections. 9th ASIS SIG/CR Classification Research Workshop, 1-28. doi:10.7152/acro.v9i1.12742

Proceedings of the 9th ASIS SIG/CR Classification Research Workshop

be able to retrieve the portion of the video that demonstrates the subject term, or emotion, specified in the search.

\section{Software designer's general remarks}

Bibliographic cataloguing schemes, such as the Dublin Core or the MARC bibliographic record cannot satisfy the indexing requirements of an image collection with multiple indexing facets. Any retrieval environment that supports the indexing of full text with SGML or XML can support the indexing and retrieval needs of a user-based image collection. The indexer defines the metadata tags needed and then enters the surrogate records with each metadata element under the appropriate tag.

An example XML record based on the La Bayadere indexing record follows:

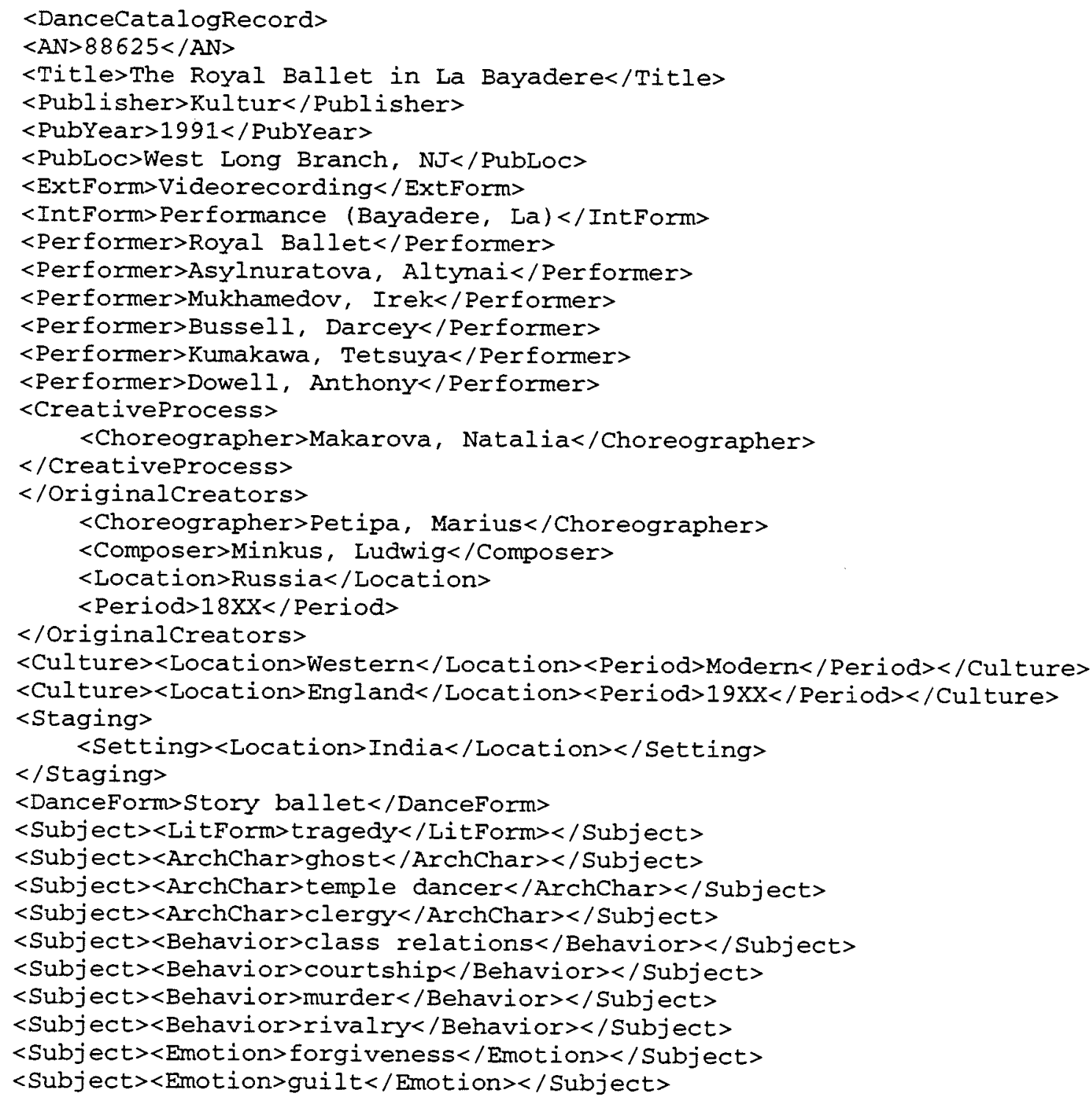




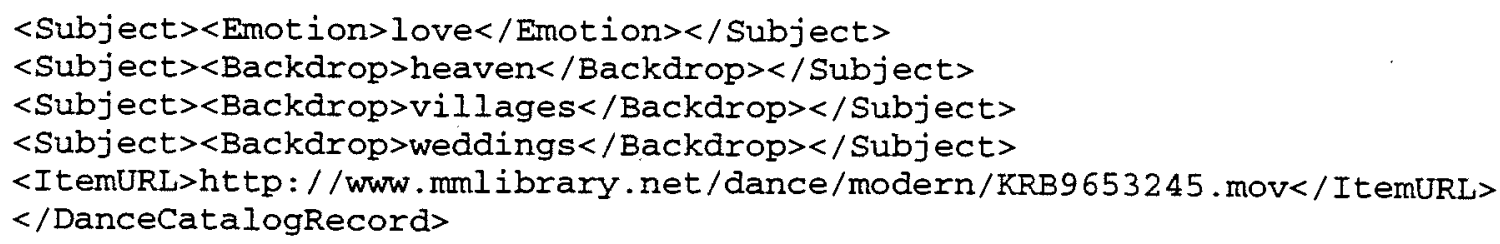

Political cartoons tend to have simpler conceptual structures than dance performances, though this in no way diminishes their cultural importance. A record for the Toles Earth Day cartoon in Figure 2, abbreviated somewhat, follows.

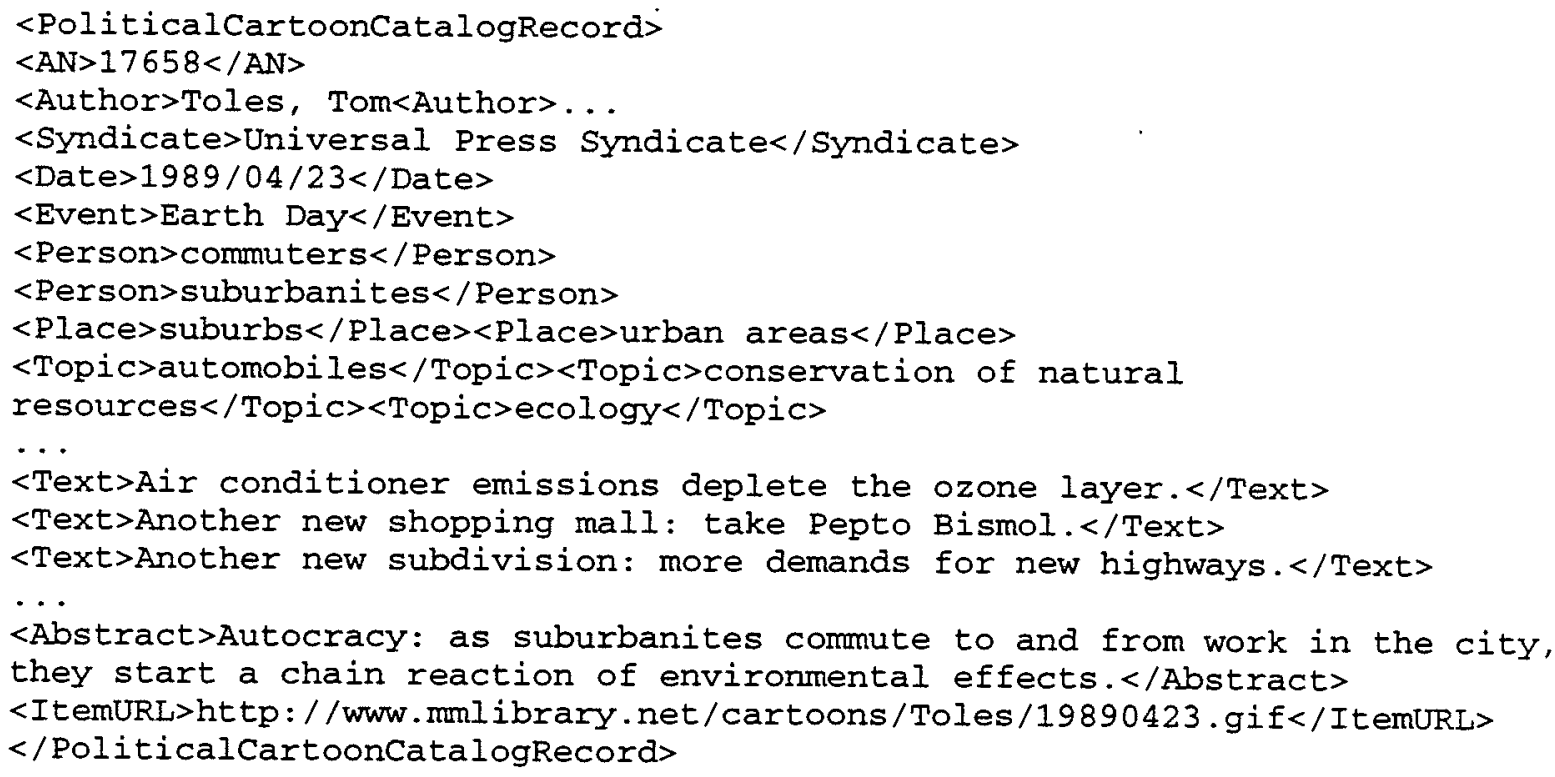

A system that would meet the general requirements outlined above is best described by illustrating hypothetical systems based on the specific requirements of the cartoon and dance image collections. Each works exactly the same except for the way they are configured; learning how to use one would allow you to use the other.

A graphical user interface (GUI) can support the entry of queries and the display of their results, the display of authority files, and the transfer of descriptors from authority file displays to the query entry area. Figure 4 shows a search window for the dance collection with queries in the top half of the window (the query entry area) and results displayed in the bottom half. The query entry area has three queries, each with a selection button to its immediate left. Clicking the selection button of a query selects it and displays its results in the bottom half of the search window. The figure shows the query $\mathbf{C L}$ Western as currently selected, with its results displayed.

The query CL Western consists of two parts, the qualifier (CL) and the search term (Western). The qualifier describes which field to search in the database, and is almost always an abbreviation for the full name of the field. CL stands for Cultural Location. So the query CL Western searches the database for records in which the cultural location is "Western". 
One way to enter a query is to type it directly into the query area of a search window.

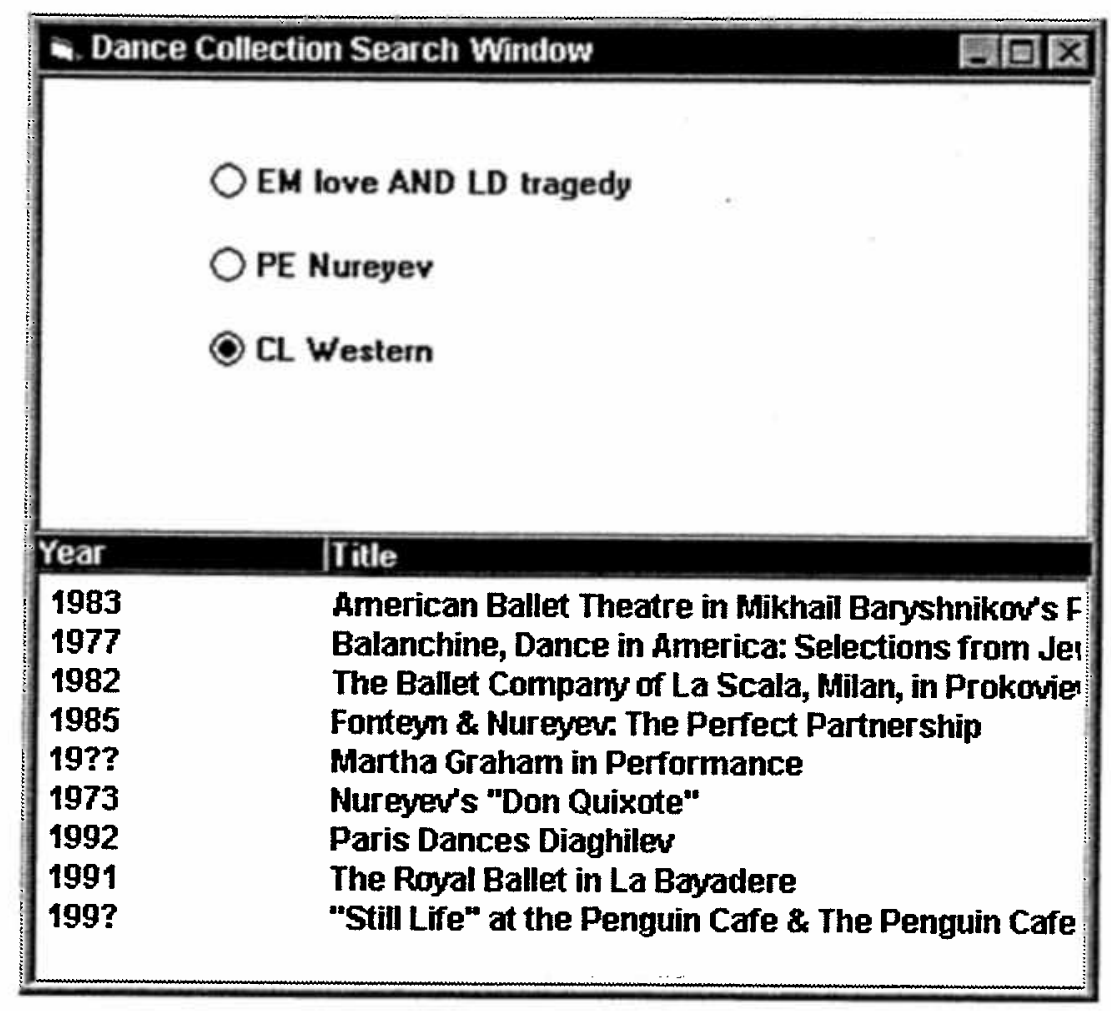

Figure 4. Dance collection search window.

A query displays the qualifiers already applied to it and gives you access to other qualifiers you may also apply to it. You may arbitrarily add or delete a query's qualifiers at any time, either by typing them as part of the query or by selecting them from menus that appear below the query. The query below shows an unqualified query as displayed in a search window. To simplify the display of individual queries, the selection buttons are not shown.

\section{Watergate tapes}

Retrieval systems should allow unqualified queries, in which case queries would simply be words or phrases which the search engine would use for either a full text search or a search on a set of default indexes, which may retrieve a large number of records from the collection. If you know which qualifiers to use, you can add them to the query, reducing its scope and therefore the number of items retrieved. However, multifaceted conceptual schema often make it difficult to remember all the qualifiers available for a given collection. Here the creative use of GUI displays can help, as illustrated in what follows. 


\section{Designer's comments on specific requirements}

Here each requirement from section IV is covered point by point, with explanations of how a GUI can satisfy it.

Requirement 1 . The search window should reflect (graphically) the facets of the scheme that were used to index the image.

A menu can provide the means by which you select qualifiers (names of facets) to use in a query. However, as traditionally implemented on OPACs, these menus force you to select the qualifier(s) before typing the search term. This is the opposite of how it ought to work. Once you have entered a search term, you ought to be able to qualify it in any number of ways without having to rekey it, or even copy-and-paste it from one place to another. It should also allow you to use abbreviated qualifier names, yet still show you the full qualifier name so that you can verify that you used the right one.

Passing the mouse over the query (known as a "mouse-over" action) causes it to display detailed information about itself. A description of the existing qualifiers, as well as a button to display the available qualifiers, appears below the query. Information about the search term appears above the query. See Figure 5, below.

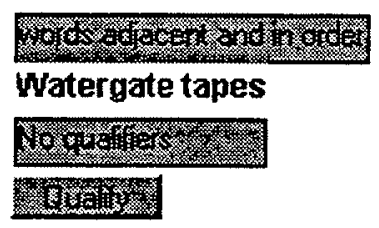

Figure 5. Cartoon query with mouse-over display.

Clicking on the "qualify" button displays the top level of qualifiers available to assign to the query. See Figure 6, below. Each qualifier is listed as a menu item with the full name of the qualifier. If the qualifier can be assigned to the query directly, its abbreviation follows. In Figure 6 , you can apply the qualifiers Subject and Visual to the query, since their respective abbreviations SU and VIS follow their names in the menu item. You cannot apply Item to the query, since it has no abbreviation, requiring you to select a qualifier from its sublist.

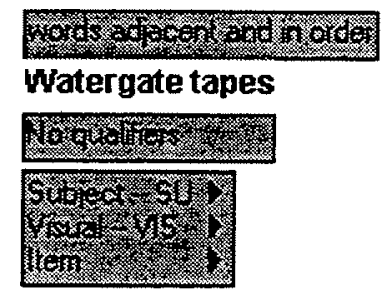

Figure 6. Cartoon query with top-level qualifier menu. 
A qualifier may have a sublist, corresponding to the conceptualization of the faceting scheme devised by the indexer. Passing the mouse over a qualifier that indicates a sublist opens that sublist. With a sublist open, you can select a qualifier on that sublist just as you can a qualifier on the main list. See Figure 7.

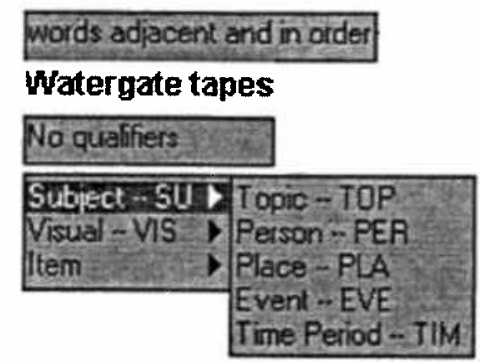

Figure 7. Query with Subject access qualifier sublist.

Clicking on "Topic" in the qualifier sublist adds that qualifier to the query, as follows.

\section{TOP Watergate tapes}

Passing the mouse over the query again shows detailed information about the query. In Figure 8, the qualifier display area shows "Topic" as the qualifier, explaining the abbreviation "TOP" that precedes the search term.

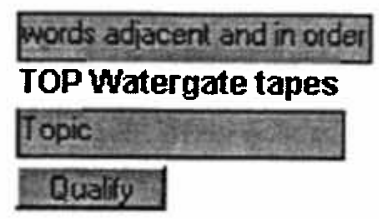

Figure 8. Cartoon query with mouse-over display showing the full name of the qualifier TOP.

You can select more qualifiers from the menus. See Figure 9.

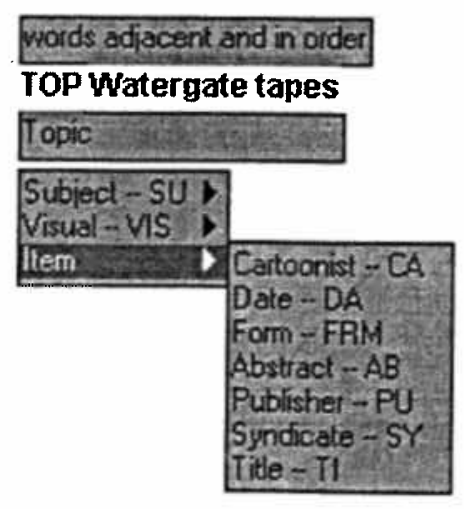

Figure 9. Cartoon query with Item qualifier sublist. 
Clicking on "Abstract" in the qualifier sublist adds it to the query as follows.

\section{TOP, AB Watergate tapes}

When a query has multiple qualifiers, each qualifier is separated by a comma. When you select multiple qualifiers from the menus, the software automatically inserts the commas for you. You can also type the qualifiers directly into the query, without using the menus, but you must then remember to type the commas. Passing the mouse over the query displays the query description area and gives you access to the qualifier menus whether you originally picked qualifiers from the menus or typed them yourself. Queries are not case sensitive, so top, ab watergate tapes is equivalent to TOP, AB Watergate tapes. Users of systems that employ the Common Command Language (Z39.58) should recognize this syntax; it is based on that same standard.

Subject access for dance materials works the same way, though the menu structures differ. Figure 10 shows some of the qualifier menu structure derived from the dance materials conceptualization.

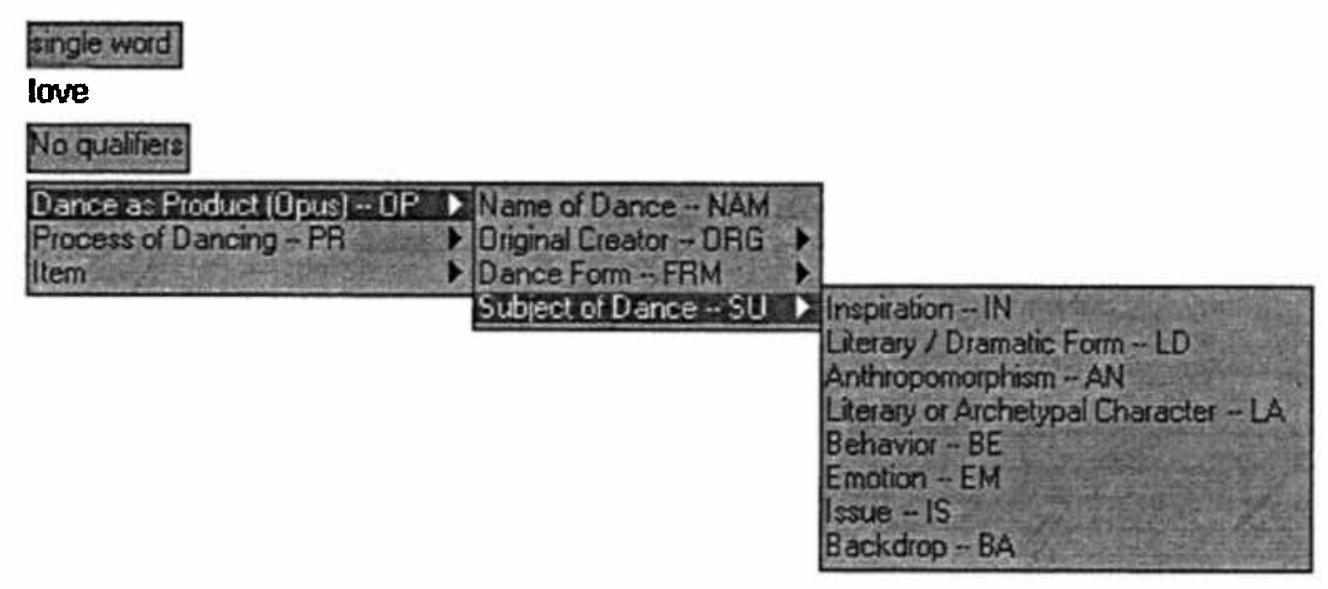

Figure 10. Dance query with Subject of Dance qualifier sub-sublist of the Opus qualifier sublist.

You can pick the Subject of Dance (SU) qualifier, covering all subject access facets, or one of the specific facets if you are sure about which one contains the search term, probably Emotion (EM) in this case. This degree of specificity of facets and the descriptors used within them, although it makes for comprehensive indexing, often presents a problem to the searcher. For this reason, to select effective search parameters you need access to authority files of descriptors used for each facet.

Requirement 2. Facets should be displayed in a form that is most appropriate to that facet (e.g., a hierarchy for a thesaurus; a zoomable timeline for a date; a navigable, zoomable map for a place).

A GUI offers many ways to activate an authority display. The mouse-over display in Figure 11 shows that the query has an Emotion qualifier. 


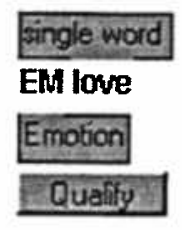

Figure 11. Dance query with mouse-over display.

Clicking on the word "Emotion" in the qualifier display opens an authority file, or thesaurus, display for the Emotion facet, showing the entry in the Emotion Authority file for the search term in the query, in this case the descriptor "love". See Figure 12. Similarly, clicking on a date or time period would display a zoomable timeline, and clicking on a geographical name would display a zoomable map.

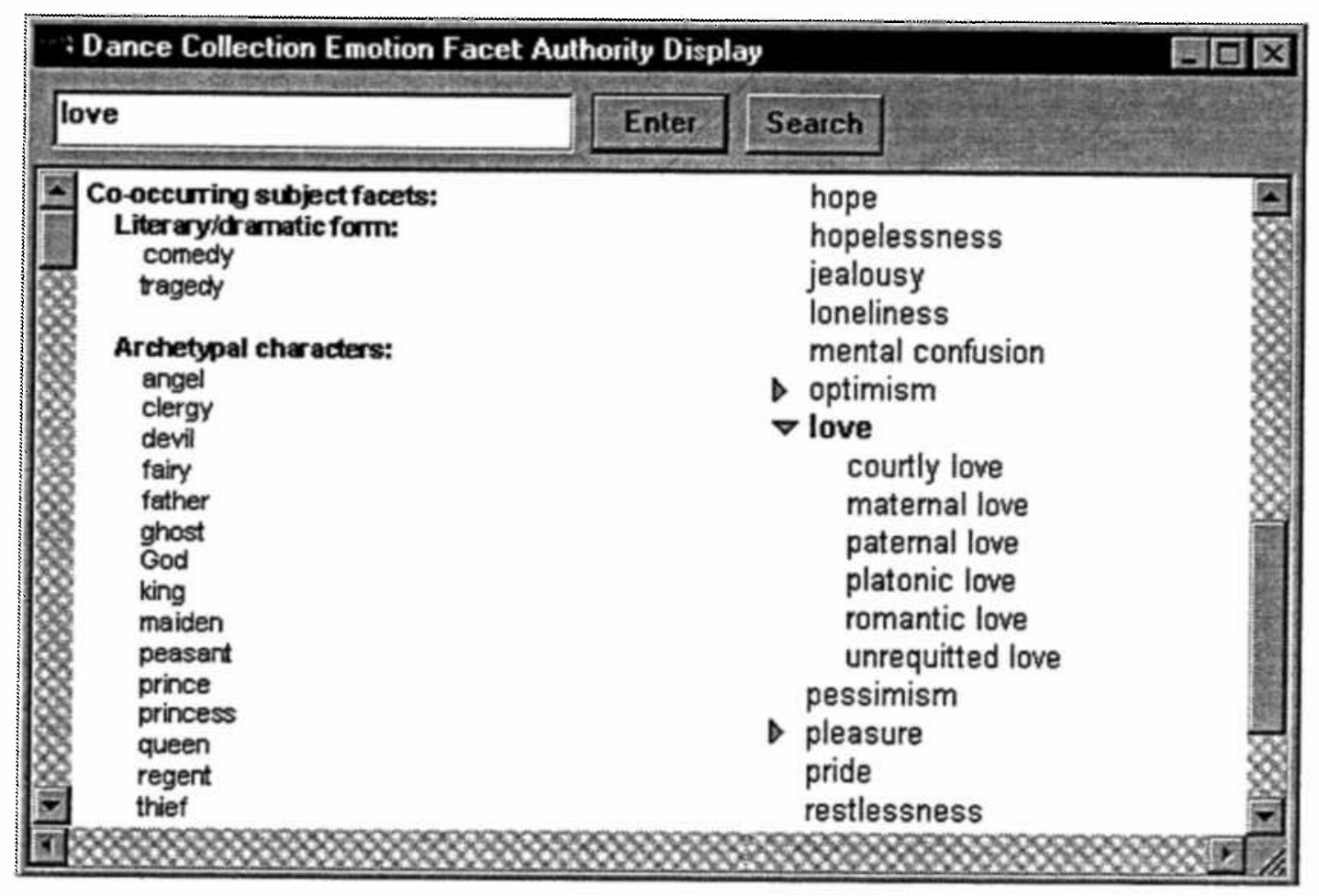

Figure 12. Emotion facet authority display for love.

A facet authority display has two columns. The left-hand column displays scope notes, related terms (if any), and descriptors from related facets that co-occur in bibliographic records. This preprocessed list allows you to see, for example, that the dance video collection contains at least one performance that portrays the emotion of love and has a ghost as an archetypal character. Not every possible facet is processed however; in this example, only the facets beneath the overarching Subject facet are processed into co-occurrence lists that appear in each other's facet authority display. There are no time or place descriptor co-occurrence listings in facets under the Subject facet, but they could be displayed as separate "limit" options in the query window. 
The right-hand column displays the facet hierarchy, with the current descriptor shown in boldface. Expansion triangles to the left of descriptors having narrower terms allow you to expand or collapse parts of the hierarchy. Each column is independently scrollable.

Requirement 3. The searcher should be able to browse the hot-linked authority files, so that any term seen could be used as an actual search term without re-keying it.

Requirement 4. For each facet, the searcher should be able to see a browse list of the thesaurus terms used in that facet, including the ability to jump to displays for related terms through hypertext.

All descriptors in the facet authority display are hypertextual. Clicking on a descriptor shows the authority display for that descriptor, along with co-occurring descriptors from other facets. Clicking on a descriptor from a different facet shows you the descriptor display for that facet. For example, clicking on the descriptor ghost in Figure 12 shows you the archetypal character facet authority display, with the archetypal character hierarchy and the list of co-occurring dramatic forms, emotions, etc.

This kind of scheme works for timeline and geographic display as well. A zoomable timeline could display a hierarchy of epochs, with expansion triangles allowing you to progressively expand and zoom in on smaller time periods. When you have a particular time period selected, the left-hand side of the display shows geographic descriptors of dance performances set during that time. Clicking on a geographic descriptor opens a zoomable map showing the region, which in turn lists timeline descriptors for performances set in that region.

Requirement 5 . The user should be able to specify more than one term to search for each facet and to combine terms from various facets easily.

You can drag-and-drop any descriptor into the query window to perform a new bibliographic search. When you drag a descriptor into the query window, the proper qualifier comes with it, and when you drop it there the new search is automatically submitted to the bibliographic server. When you drag a descriptor into the query window, you can either drop it by itself to form a new query, or drop it into another query to form a Boolean query. For example, dropping the emotion descriptor love into the query window forms the query shown in Figure 11. Dropping the literary/dramatic form descriptor tragedy by itself into the query window forms the query

\section{LD tragedy}

However, dragging it into the proximity of an existing query causes the existing query to react so as to display the default form of the combined queries. 
En love

a.

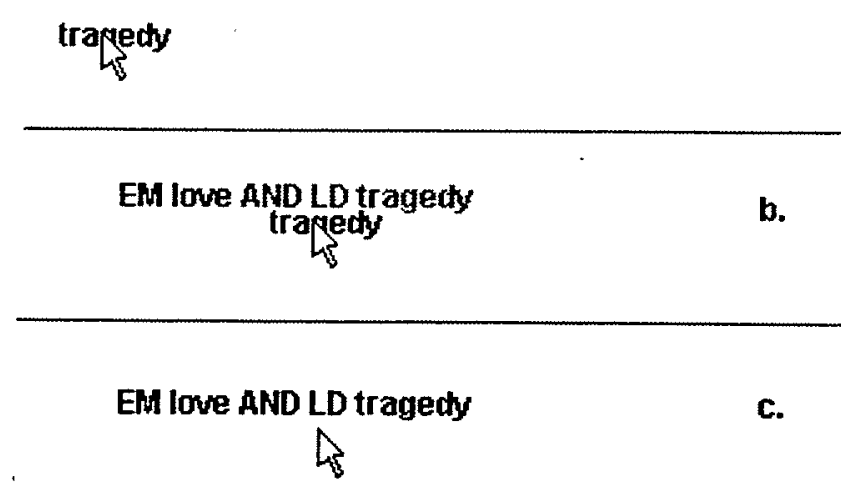

Figure 13. Combining two facet descriptors into a Boolean AND query.

Figure 13 shows three stages of combining two facet descriptors into a Boolean query. In (a), you drag the descriptor tragedy from the facet authority display into the query window. As long as you do not bring the descriptor close to another query, it will act as a simple query when you drop it into the query area. Part (b) shows what happens when you bring the descriptor close to an existing query: while you keep the mouse button down, the existing query "pencils in" the change that will occur if you release the mouse button right then. At this point you can still drag the descriptor away from the existing query (at which point the existing query erases the penciled-in part) to combine it with other queries or use it to form a new query. Part (c) shows what happens when you release the mouse button at the point shown in (b).

The structure that an existing query pencils in during a descriptor drag-over depends on the structure and qualifiers already in the query and the qualifier attached to the dragged descriptor. The particular situation in Figure 13 has an existing query with one search term and qualifier and a dragged descriptor with a different qualifier; the default behavior in this case is to combine the two with a Boolean AND. However, if the two have the same qualifier the existing query pencils in an OR between the two search terms and a factoring of the qualifier. For example, if you drag the emotion descriptor mental confusion from the facet authority display close to the existing query in Figure 13 (a), the pencilling-in would look like Figure 14.

\section{EH (love OR mental confusion) mental cpnfusion}

Figure 14. Combining two facet descriptors into a Boolean OR query.

This can work with arbitrarily complex queries. Taking the existing query from Figure 13(c), the mental confusion pencilling-in would look like Figure 15. 


\section{EH (love OP mental comfusion) AND LD tragethy mentai cpmfusion}

Figure 15. Combining a facet descriptor with an existing Boolean query.

This makes it very easy to construct Boolean queries in conjunctive normal form, commonly used by searchers when querying more than one field while hedging each with more than one search term. Searchers often have trouble writing the correct Boolean syntax for these types of queries, and experienced searchers, though they have no difficulty understanding the syntax, still find it tedious to construct when they have to type it all out. Even novice users who do not really understand Boolean syntax, and would have difficulty writing a correct Boolean query, can construct useful queries with the default user interface behavior illustrated here.

However you construct a query, you can always edit it directly in the query area, just as you edit text in a GUI-based word processor. If you are so inclined, you can even type complex Boolean queries without resorting to the authority files or any of the on-screen qualifier and descriptor selection features described here. But anytime you want to use them they are there for you, no matter what method you used to construct the query.

Requirement 6. Most important for searching a faceted database, as the user specifies and respecifies the search terms they require from multiple facets, the search results should be easy to compare.

The windowed search interfaces proposed here allows you to create multiple instances of search windows (like the one shown in Figure 4) which you can juxtapose on the computer screen to compare results from different queries.

Requirement 7. If the hoped-for term is not in the thesaurus, the interface should guide the searcher to an approximate synonym that is in the thesaurus. Back-up free-text indexes for the collection or synonym dictionaries should be accessible at this stage of the search, without disrupting the search's line of inquiry.

The thesaurus display can follow Use references in the thesaurus to take you from an access term to the preferred synonym.

In a similar way, you can have trouble finding an access point into a thesaurus display if you do not know the actual vocabulary. Keyword in Context (KWIC) databases are easily derived from bibliographic and authority files, and their use in retrieval GUIs enhance both the navigation of facet authority files and the construction of bibliographic queries.

A KWIC display works by showing you phrases that match the context of what you type. The phrases it displays come from the part of the collection that you are searching. Figure 16 shows the keyword love typed into the facet authority display, with matching keywords and descriptors under behavior, emotion, etc. When you use the KWIC display with the facet authority display, clicking on a descriptor in the KWIC display automatically displays the record 
for that descriptor in the facet authority display. For example, clicking on the descriptor love under Emotion in the KWIC display shows the record for love in the emotion facet, as in Figure 12.

\begin{tabular}{|l|}
\hline Keyword in Contest \\
\hline Keywords \\
\hline love \\
lovebird \\
loved \\
loveless \\
lovelock \\
lovely \\
loveliness \\
loves \\
\hline Behavior \\
\hline first loves \\
love-hate relationships \\
love in adolescence \\
love in old age \\
love loss \\
\hline Emotion \\
\hline love \\
courthy love \\
maternal love \\
\hline
\end{tabular}

Figure 16. Keyword-In-Context (KWIC) display for the keyword love.

For authority files, KWIC databases include UF references, so you can access the preferred term in a facet by clicking on its UF reference in the KWIC display.

Requirement 8 . If no terms satisfy, the searcher should have a ready mechanism available for suggesting the term to the indexer. This "email" feature helps the indexer / lexicographer understand the research needs for which the collection is being used and to keep up-to-date on searchers' terms.

The best mechanism for this now is regular email. If you do not have convenient access to email when using the search interface, a "feedback" window could be improvised that channels typed comments to the indexer's email account.

Requirement 9. Current catalog records for visual materials may provide some descriptive information for items. It would be useful if these records already in existence either from OCLC, or the New York Public Library or elsewhere, could be easily attached to our records with the new indexing terms.

You should not have to know that one part of a record came from, for example, OCLC or the New York Public Library if this is not important to you. The actual copy-cataloguing involved in augmenting existing records with additional indexing terms goes on in the back rooms of libraries, but you should still be able to find out where a field or descriptor came from. 
Requirement 10. As the Internet will be the way to access these collections, we need to access the image or, in the case of a video, perhaps access a part of the total production. For cartoons, the searcher should be able to retrieve the cartoon on-line during the search. For videos, the searcher should be able to retrieve the portion of the video that demonstrates the subject term, or emotion, specified in the search.

The multimedia capabilities of PCs and the high bandwidth now available over networks allow online access to multimedia content. The catalog record for the item can point to its URL (see the tags ItemURL and their content in the sample catalog records earlier in this article), so that the retrieval client can use a standard World Wide Web connection to download it. Appropriate viewing software either incorporated into the retrieval client or launched by it can display the file.

\section{Conclusion}

From the interaction exhibited here between two index creators and a system designer we have demonstrated how specialized arrangements such as faceted vocabulary schemes can be accommodated by an adaptable, generalized retrieval scheme as long as the user's needs are kept uppermost and the indexing records and authority files allow for flexible use.

\section{References}

Bovey, J.D. (1993). "A Graphical Retrieval System”. Journal of Information Science, 19, 179188.

Bovey, J.D. (1995). "Building a Thesaurus for a Collection of Cartoon Drawings". Journal of Information Science, 21(2), 115-122.

Chapple-Sokol, Angie. (1996). “Indexing Editorial Cartoons”. Special Libraries, 21-31.

Cochrane, P., and Johnson, E.H. (1996). "Visual Dewey: DDC in a Hypertextual Browser for the Library User." In Advances in Knowledge Organization, Vol. 5 (pp. 95-106). Washington, D.C.: International Society for Knowledge Organization.

Croghan, A. (1968). Classification of the Performing Arts. London: North Western Polytechnic.

Fischer, R.A. (1996). Them Damned Pictures: Explorations in American Political Cartoon Art. North Haven, CT: Archon Books.

Hanna, J. L. (1970). "Dance and the Social Sciences: An Escalated Vision". In M. Haberman and T.G. Meisel (Eds.), Dance: An Art in Academe. New York: Teachers College Press. 
Hess, S., and S. Northrop. (1996). Drawn \& Quartered: The History of American Political Cartoons. Montgomery, AL: Elliot \& Clark.

Johnson, E.H. (1995). "Extending an Interactive Thesaurus by Dragging," Siglink Newsletter, 4(2), 16-17.

Johnson, E.H. (1998). "The Search Document Paradigm for Information Retrieval". Unpublished manuscript, submitted to International Journal on Digital Libraries: Special Issue on User Interfaces for Digital Libraries.

Johnson, E.H. “An Object-Enhanced User Interface for Information Retrieval”. Unpublished manuscript, to appear in Science \& Technology Libraries: Special Issue on Digital Libraries.

Johnson, E.H., and Cochrane, P. (1995). "A Hypertextual Interface for a Searcher's Thesaurus." Paper presented at Digital Libraries '95, Austin, June 11-13, 1995.

Lavender, L. (1995). "Understanding Interpretation.” Dance Research Journal, 27(2), 25-33.

Lent, J.A., compiler. (1994). Comic Books and Comic Strips in the United States: An International Bibliography. Westport, CT: Greenwood Press.

Ludden, B. (1968). "The Dance Classification System of the Julliard School Library". Theatre Documentation, 21-29.

National Information Standards Organization. (1992). Common Command Language for Online Interactive Information Retrieval. Bethesda, MD: NISO Press.

Satin, A.D. (1985). A Doonesbury Index: An Index to the Syndicated Daily Newspaper Strip "Doonesbury" by G.B. Trudeau, 1970-1983. Metuchen, NJ: Scarecrow Press.

Schatz, B., Johnson, E.H., Cochrane, P., and Chen, H. (1996). "Interactive Term Suggestion for Users of Digital Libraries: Using Subject Thesauri and Co-Occurrence Lists for Information Retrieval". Paper presented at the Digital Libraries '96: 1st ACM International Conference on Research and Development in Digital Libraries, Bethesda, MD, March 20-23, 1996. 
Barnes, B., Johnson, E., Young, J., \& Cochrane, P. (1998). Generalized software requirements to access thesauri and classification schemes for user-based image collections. 9th ASIS SIG/CR Classification Research Workshop, 1-28. doi:10.7152/acro.v9i1.12742

Proceedings of the 9th ASIS SIG/CR Classification Research Workshop 